\title{
Clinical status of comorbid bipolar disorder and borderline personality disorder
}

\author{
Gordon Parker, Adam Bayes, Georgia Mcclure, Yolanda Romàn Ruiz del Moral
} and Janine Stevenson

\section{Background}

The status and differentiation of comorbid borderline personality disorder and bipolar disorder is worthy of clarification.

\section{Aims \\ To determine whether comorbid borderline personality disorder and bipolar disorder are interdependent or independent conditions.}

\section{Method}

We interviewed patients diagnosed with either a borderline personality disorder and/or a bipolar condition.

\section{Results}

Analyses of participants grouped by DSM diagnoses established that those with comorbid conditions scored similarly to those with a borderline personality disorder alone on all key variables (i.e. gender, severity of borderline personality scores, developmental stressors, illness correlates, self-injurious behaviour rates) and differed from those with a bipolar disorder alone on nearly all non-bipolar item variables. Similar findings were returned for groups defined by clinical diagnoses.

\section{Conclusions}

Comorbid bipolar disorder and borderline personality disorder is consistent with the formal definition of comorbidity in that, while coterminous, individuals meeting such criteria have features of two independent conditions.

\section{Declaration of interest}

None.

\section{Copyright and usage}

(c) The Royal college of Psychiatrists 2016.
It is well recognised that bipolar disorder is extremely impairing and Zimmerman and colleagues ${ }^{1}$ have shown that the psychosocial morbidity associated with borderline personality disorder is as great or greater than for bipolar disorder. Diagnostic differentiation of a bipolar disorder from a borderline personality disorder is commonly judged as difficult. ${ }^{2-9}$ In a recent study, ${ }^{10}$ we undertook multivariate analyses of candidate variables (for example sociodemographic, mood state, family history, developmental factors, personality style, illness correlates) that might best differentiate those with 'pure' bipolar disorder or borderline personality disorder conditions, and with our refined set of variables differentiating with more than $90 \%$ accuracy. We now address a related 'real-world' clinical issue in seeking to differentiate those comorbid for both conditions from those with a sole bipolar disorder or a sole borderline personality disorder, being aware of only one such related study that compared those with bipolar II depression with those with comorbid borderline personality disorder. ${ }^{11}$ Our principal analyses involve use of DSM criteria alone. Such a strategy, however, risks creating artefactual 'comorbidity' if there are similar criteria for each condition, a salient concern when bipolar disorder and borderline personality disorder are both defined in DSM as conditions marked by affective instability, impulsivity, an unstable sense of self and problems in relationships. To obviate this risk, subsidiary analyses used clinician-judged diagnoses. Clinician assessment has the potential to overcome artefactual comorbidity emerging from similar formalised criteria as assessment can range over a wider set of candidate variables, including the interview behaviour of the participant, and generally operates to a rule of parsimony (i.e. choice of only one diagnosis). Second, although one of our clinical diagnostic strategies respected DSM bipolar disorder duration criteria (i.e. hypomanic episodes lasting 4 or more days for bipolar II disorder and manic episodes lasting 7 or more days for bipolar I disorder), the other clinical diagnostic strategy ignored duration criteria in light of reviewed studies ${ }^{12}$ suggesting that DSM duration criteria may be too stringent. Thus, our study was designed to determine how those with comorbid bipolar disorder/borderline personality disorder might best be differentiated from those with either a pure bipolar disorder or a pure borderline personality disorder, and to examine the impact of differing diagnostic strategies on such differentiation.

\section{Method}

\section{Recruitment and measures}

As the study design has already been reported ${ }^{10}$ here we summarise the key details. We sought participants aged 18 years and older who had received a previous diagnosis of type I or II bipolar disorder or borderline personality disorder, recruiting participants from several clinical services, as well as via advertisements in newspapers and our institute's website. Site ethical approval was ratified by the University of New South Wales Human Research Ethics Committee and written consent was obtained from all participants. Exclusion criteria included English language limitations, psychotic or significant organic features and current substance dependence.

Potential participants were provided with a booklet assessing sociodemographic, mood history, family history details, treatment information and a 145-item self-report measure of ongoing personality style weighted to borderline descriptors, as well as childhood experiences and self-destructive behaviour. Participants also completed the Measure of Parental Style (MOPS) ${ }^{13}$ in relation to each parent as remembered over the first 16 years of their life, generating MOPS parental indifference, overcontrol and abuse scale scores.

A detailed semi-structured interview was undertaken by a psychiatrist (A.B.). Clinical information included assessing for 
childhood sexual abuse, 'developmental trauma' other than childhood sexual abuse, whether one or both parents were distant or rejecting and whether the participant had experienced depersonalisation in childhood. The diagnostic aim of the interview was to establish the presence (or absence) of a type I or II bipolar disorder and/or borderline personality disorder diagnosis. Study 'DSM' diagnoses respected DSM-IV criteria ${ }^{14}$ for both conditions. In practice, a DSM-IV type I or II bipolar disorder diagnosis was determined by administering the Mini International Neuropsychiatric Index (MINI) ${ }^{15}$ to all participants. A DSM-IV borderline personality disorder diagnosis of borderline personality disorder was generated after administering the Diagnostic Interview for Personality Disorders IV (DIPD-IV) Borderline Personality Disorder section ${ }^{16}$ to all interviewees, and those scoring two on five or more of the nine criteria received a DSM-IV borderline personality disorder diagnosis.

Our 'clinical diagnoses' were generated from the comprehensive interview. If the psychiatrist judged a bipolar disorder as present with that decision respecting DSM-IV symptom criteria but also considering general history data and any referrer information diagnostic subtyping and allocation occurred. A clinical diagnosis of a bipolar I disorder was assigned simply on the basis of a diagnosed bipolar disorder participant describing psychotic features during previous manic states, whereas a clinical bipolar II disorder diagnosis required the absence of psychotic features when hypomanic. A borderline personality disorder 'clinical' diagnosis was determined by the clinician weighting DSM-IV criteria but also considering any corroborative referrer information and interview nuances. We generated two clinical bipolar disorder subgroups - a 'clinical strict diagnosis' group comprised those meeting DSM duration criteria for hypomania or mania, and a 'clinical extended diagnosis' group that also included those reporting briefer hypo/manic episodes than stipulated by DSM.

\section{Data analysis}

Our 145-item booklet measure contained 113 personality items weighted to a borderline personality style. Seeking to optimise the most discriminating items, we refined the set by analysing for differences on item scores across the three DSM diagnostic groups (i.e. bipolar disorder, borderline personality disorder and bipolar disorder/borderline personality disorder - the last now termed 'comorbid'), and quantified the extent to which there were group differences (by one-way ANOVAs and an imposed significance level of $P<0.001)$. Inspection of the 25 most discriminating items (Appendix 1) indicates that they capture cognitions commonly accepted as integral to a borderline personality style. Item responses were summed to generate a total borderline personality score for study analyses.

One-way between-groups analysis of variance explored the relationship between DSM diagnostic allocation (bipolar disorder, borderline personality disorder, comorbid) and continuous variables such as age. Chi-squared tests of independence quantified associations between diagnostic allocation and categorical variables (for example admission to hospital). Log-linear contrasts were conducted on principal variables identified as showing group differences. Such contrast analyses handled dimensional variables (for example borderline personality measure scores) by calculating the median score on that variable for the entire sample, and then creating dichotomous categories for those above and below the median. Our principal study analyses focus on those diagnosed by DSM criteria, with the other two diagnostic strategies being supplementary. All statistical tests were performed using SPSS, Version 22.

\section{Results}

After excluding those who failed to meet relevant diagnostic criteria for either condition, our provisional sample comprised 190 individuals who completed both the self-report measure and diagnostic interview and received a DSM bipolar disorder and/or a DSM borderline personality disorder diagnosis from the assessing clinician. Of the 137 receiving a DSM-IV bipolar disorder diagnosis (alone or comorbid), 11 (8\%) met bipolar I and 126 (92\%) met bipolar II disorder criteria. As illustrated in Fig. 1, rates of comorbid assignment were highest $(28 \%)$ in relation to DSM diagnoses and 13\% for those receiving a bipolar disorder clinical strict diagnosis and $14 \%$ for those receiving a bipolar disorder clinical extended diagnosis. Although 53 participants were assigned a borderline personality disorder diagnosis across the two diagnostic systems, they were not the same 53 individuals. Thus, of the 53 participants assigned a borderline personality disorder diagnosis by DSM criteria, only 42 received a clinical borderline personality disorder diagnosis.

\section{Univariate analyses of group differences in those assigned by DSM criteria}

Univariate analyses compared those assigned by DSM diagnoses to a bipolar disorder, borderline personality disorder or comorbid category, with respective group numbers of 83, 53 and 54 respectively. Mean ages across groups were comparable (bipolar disorder 35.4; borderline personality disorder 32.9, bipolar disorder/borderline personality disorder 36.2 years; $F=0.97$ ). Imposing a significance level of $P<0.01$, those in the three groups did not differ by marital status, highest level of education achieved, employment status, rates of a first-degree relative having depression, bipolar disorder or having died by suicide, age at first depressive episode, average length of depressive episodes, number of suicide attempts, age of initial suicide attempt, number of any self-harm episodes, receipt of an antidepressant, nor in perceived effectiveness of any trialled antidepressant medication.

Seven hypo/manic symptoms are listed in the MINI to diagnose a hypomanic or manic episode. The mean number of hypomanic/manic symptoms for those with a DSM diagnosis of borderline personality disorder was 3.3 (s.d. $=1.6$ ) as against 5.6 (s.d.=1.6) for the participants with bipolar disorder and 5.9 (s.d.=1.0) for the participants with comorbid bipolar disorder/ borderline personality disorder, with the group difference statistically significant $(F(2,185)=66.8, \quad P<0.001)$. Post hoc comparisons using the Tukey HSD (honest significant difference) test indicated that the mean number of hypo/manic symptoms for those with a DSM borderline personality disorder diagnosis was significantly lower than for those diagnosed with bipolar disorder alone or a comorbid diagnosis, but did not differ between the latter two conditions.

Online Table DS1 lists variables evidencing significant differences across the three groups. The bipolar disorder group had a lower rate of women compared with the two other groups that had comparable high female preponderance rates. In terms of developmental factors (childhood sexual abuse, developmental trauma, depersonalisation in childhood, viewing one or both parents as distant or rejecting), the prevalence rates were lowest in the bipolar disorder group when three-group comparative analyses were undertaken. Scale scores on the MOPS measure across the three diagnostic groups showed a similar pattern, with bipolar disorder participants reporting the lowest rates of exposure to parental indifference, overcontrol and abuse, albeit with the trend for maternal indifference being the only MOPS scale lacking a significant group difference. The bipolar disorder 

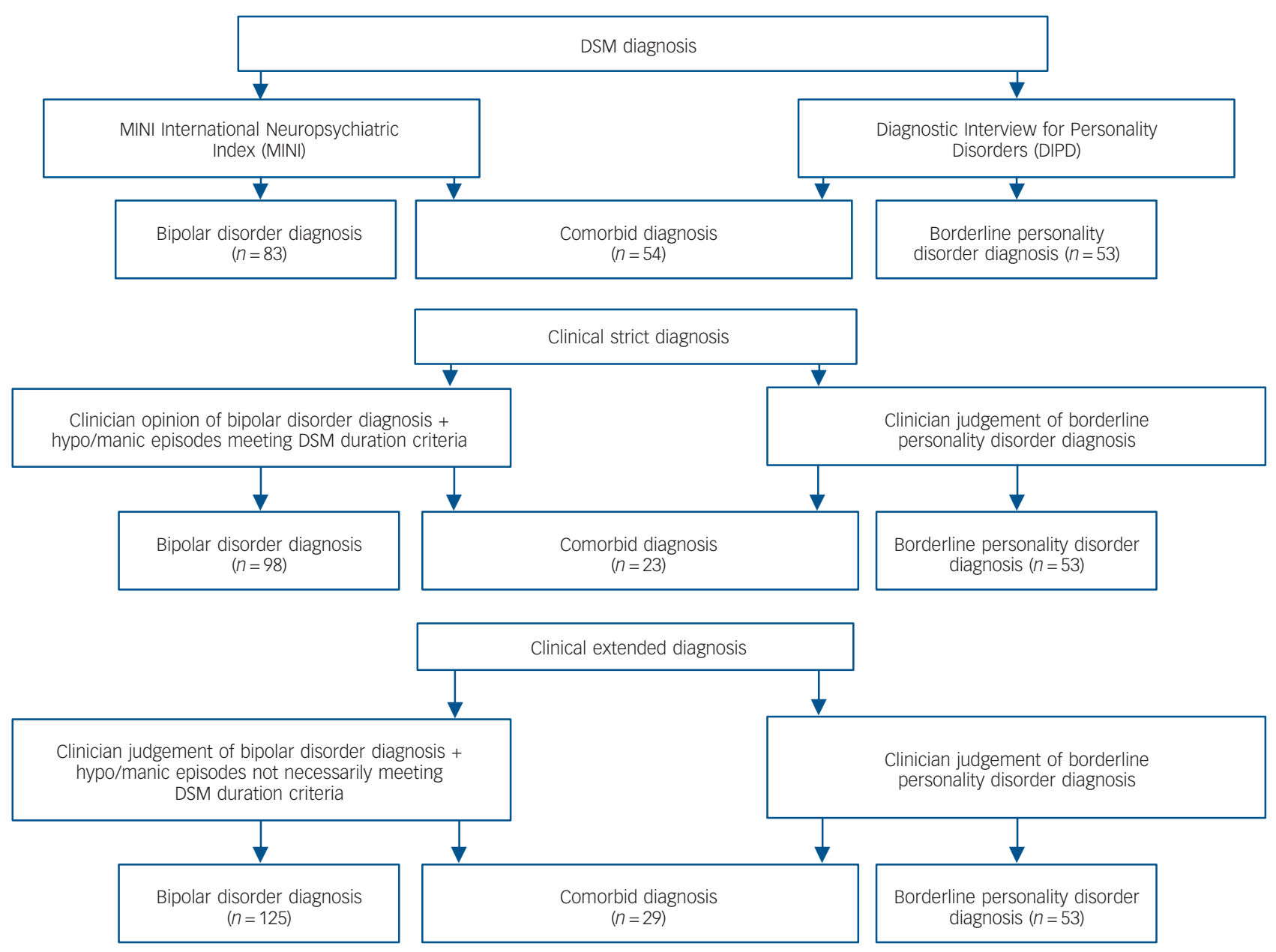

Fig. 1 Flow chart of diagnostic classification systems.

group returned distinctly lower total borderline personality measure scores than the comparably high scores in the two other groups. There was a high rate of trialling a mood stabiliser in the bipolar disorder group compared with lower rates in the comorbid group and an even lower rate in the borderline personality disorder group. Rates of judged effectiveness of any trialled mood stabiliser showed a similar linear pattern across the three groups. Rates of being admitted to hospital for a hypo/manic episode demonstrated a counterintuitive finding with the highest rate reported by the comorbid group and very low rates in the two other groups. Admission to hospital for a depressive episode had the lowest rate in the bipolar disorder group and the highest in the comorbid group. Hospital admission for a self-harm episode was lowest in the bipolar disorder group and higher and comparable in the two other groups. Lifetime rates of suicide and of self-harm attempts were similarly lowest in the bipolar disorder group and high and comparable in the borderline personality disorder and comorbid groups.

We undertook a series of log-linear analyses examining for differences between paired groups (i.e. bipolar disorder $v$. comorbid, borderline personality disorder $v$. comorbid, and bipolar disorder $v$. borderline personality disorder) for key clinical (apart from illness course) variables and which had shown group differences in our online Table DS1 analyses. Tables 1-3 report such analyses (quantified with the Wald statistic) and with the first two columns reporting results in relation to DSM assignment. Across the fourteen variables, a clear and quite striking pattern is evident. The bipolar disorder $v$. comorbid analyses were all significant at $P<0.02$, apart from maternal MOPS indifference scores. Similarly, most of the analyses comparing bipolar disorder $v$. borderline personality disorder groups were significant $(P<0.05)$ apart from those contrasting maternal MOPS indifference and overcontrol scores. Finally, none of the analyses comparing the borderline personality disorder $v$. comorbid groups were significant, nor did they show any trends for differences. In essence, the borderline personality disorder and comorbid groups were comparable on nearly all principal study variables.

\section{Analyses of group differences in those assigned by clinical strict diagnosis criteria}

Compared with DSM assignment, the clinician assigned a bipolar disorder clinical strict diagnosis (i.e. respecting DSM duration criteria) to more participants (98 v. 83) and a comorbid diagnosis to fewer (23v. 54). Imposing a significance level of $P<0.01$, mean ages for those in the three groups were comparable $(F=0.18)$ and the overall pattern of scores and distributions across the three groups was comparable with findings detailed in relation to DSM diagnosis, apart from marital (fewer married participants with borderline personality disorder) and employment status (more employed participants with bipolar disorder), age at first depressive episode (higher in the bipolar disorder group), average length of depressive episodes (longest in the borderline personality disorder group), and number of self-harm incidents (most reported by the comorbid group, followed by the borderline personality disorder group and, in turn, the bipolar disorder group). The mean number of DSM listed hypomanic or manic 
symptoms differed $(F=61.9, P<0.001)$, being lowest for the borderline personality disorder group (3.4) and higher for the bipolar disorder (5.6) and comorbid (6.2) groups.

Tables 1-3 report the log-linear analyses in relation to clinical strict diagnoses. The overall pattern was similar to the DSM analyses but not quite so pristine. Specifically, 8 of the 14 bipolar disorder $v$. comorbid comparisons were significant at the $P<0.05$ level, 12 of the 14 bipolar disorder $v$. borderline personality disorder comparisons were significant and, of central importance, none of the borderline personality disorder $v$. comorbid comparisons were significant nor showed any trend for significance.

\section{Analyses of group differences in those assigned by clinical extended diagnosis criteria}

Compared with DSM assignment, a bipolar disorder clinical extended diagnosis (i.e. not imposing DSM duration criteria) was received by a larger number of participants $(125 v .83)$ and a comorbid diagnosis by fewer participants $(29 v .54)$. Mean ages for those in the three groups were comparable $(F=0.88)$ and the overall pattern of scores and distributions across the three groups were similar to DSM diagnostic findings, apart from marital (those in the bipolar disorder group were most likely to be married) and employment status (the borderline personality

Table 1 Log-odds for three diagnostic classification systems for age and developmental factors

\begin{tabular}{|c|c|c|c|c|c|c|}
\hline & \multicolumn{2}{|c|}{ DSM diagnosis } & \multicolumn{2}{|c|}{ Clinical strict diagnosis } & \multicolumn{2}{|c|}{ Clinical extended diagnosis } \\
\hline & $\begin{array}{l}\text { Log-odds ratio } \\
\text { (Wald) }\end{array}$ & $P$ & $\begin{array}{l}\text { Log-odds ratio } \\
\text { (Wald) }\end{array}$ & $P$ & $\begin{array}{l}\text { Log-odds ratio } \\
\text { (Wald) }\end{array}$ & $P$ \\
\hline \multicolumn{7}{|l|}{ Gender } \\
\hline Bipolar disorder v. comorbid & 9.78 & 0.002 & 5.56 & 0.018 & 7.45 & 0.006 \\
\hline Borderline personality disorder $v$. comorbid & 0.26 & 0.614 & 0.39 & 0.532 & 0.07 & 0.793 \\
\hline Bipolar disorder $v$. borderline personality disorder & 14.49 & $<0.001$ & 14.95 & $<0.001$ & 14.09 & $<0.001$ \\
\hline \multicolumn{7}{|l|}{ Childhood sexual abuse } \\
\hline Bipolar disorder v. comorbid & 20.54 & $<0.001$ & 10.36 & $<0.001$ & 12.12 & $<0.001$ \\
\hline Borderline personality disorder $v$. comorbid & 0.62 & 0.432 & 0.10 & 0.747 & 0.36 & 0.547 \\
\hline Bipolar disorder v. borderline personality disorder & 14.23 & $<0.001$ & 20.06 & $<0.001$ & 23.98 & $<0.001$ \\
\hline \multicolumn{7}{|l|}{ Developmental trauma } \\
\hline Bipolar disorder v. comorbid & 12.51 & $<0.001$ & 3.53 & 0.060 & 6.24 & 0.013 \\
\hline Borderline personality disorder $v$. comorbid & 0.02 & 0.889 & 1.14 & 0.286 & 1.06 & 0.302 \\
\hline Bipolar disorder $v$. borderline personality disorder & 11.31 & $<0.001$ & 14.85 & $<0.001$ & 18.51 & $<0.001$ \\
\hline \multicolumn{7}{|l|}{ Depersonalisation } \\
\hline Bipolar disorder v. comorbid & 10.99 & $<0.001$ & 15.79 & $<0.001$ & 19.03 & $<0.001$ \\
\hline Borderline personality disorder $v$. comorbid & 0.95 & 0.330 & 0.16 & 0.286 & 0.37 & 0.541 \\
\hline Bipolar disorder $v$. borderline personality disorder & 11.31 & $<0.001$ & 30.65 & $<0.001$ & 34.93 & $<0.001$ \\
\hline \multicolumn{7}{|l|}{ Parent distant or rejecting } \\
\hline Bipolar disorder v. comorbid & 14.44 & $<0.001$ & 8.09 & 0.004 & 11.15 & $<0.001$ \\
\hline Borderline personality disorder $v$. comorbid & 0.02 & 0.888 & 0.07 & 0.793 & 0.02 & 0.891 \\
\hline Bipolar disorder $v$. borderline personality disorder & 13.62 & $<0.001$ & 14.02 & $<0.001$ & 17.59 & $<0.001$ \\
\hline
\end{tabular}

\section{Table 2 Log-odds for three diagnostic classification systems for Measure of Parental Style (MOPS) items}

\begin{tabular}{|c|c|c|c|c|c|c|}
\hline \multirow[b]{2}{*}{ MOPS items } & \multicolumn{2}{|c|}{ DSM diagnosis } & \multicolumn{2}{|c|}{ Clinical strict diagnosis } & \multicolumn{2}{|c|}{ Clinical extended diagnosis } \\
\hline & $\begin{array}{l}\text { Log-odds ratio } \\
\text { (Wald) }\end{array}$ & $P$ & $\begin{array}{l}\text { Log-odds ratio } \\
\text { (Wald) }\end{array}$ & $P$ & $\begin{array}{l}\text { Log-odds ratio } \\
\text { (Wald) }\end{array}$ & $P$ \\
\hline \multicolumn{7}{|l|}{ Maternal indifference } \\
\hline Bipolar disorder v. comorbid & 0.546 & 0.460 & 3.70 & 0.055 & 2.17 & 0.139 \\
\hline Borderline personality disorder $v$. comorbid & 0.38 & 0.537 & 0.08 & 0.772 & 0.17 & 0.679 \\
\hline Bipolar disorder $v$. borderline personality disorder & 2.01 & 0.157 & 4.84 & 0.028 & 5.49 & 0.019 \\
\hline \multicolumn{7}{|l|}{ Maternal abuse } \\
\hline Bipolar disorder v. comorbid & 14.72 & $<0.001$ & 2.71 & 0.100 & 2.38 & 0.123 \\
\hline Borderline personality disorder $v$. comorbid & 2.42 & 0.120 & 0.46 & 0.498 & 1.61 & 0.205 \\
\hline Bipolar disorder $v$. borderline personality disorder & 5.23 & 0.022 & 9.44 & 0.002 & 12.15 & $<0.001$ \\
\hline \multicolumn{7}{|l|}{ Maternal overcontrol } \\
\hline Bipolar disorder v. comorbid & 9.12 & 0.003 & 2.80 & 0.094 & 2.31 & 0.129 \\
\hline Borderline personality disorder v. comorbid & 1.79 & 0.182 & 0.06 & 0.813 & 0.23 & 0.635 \\
\hline Bipolar disorder v. borderline personality disorder & 2.65 & 0.104 & 3.78 & 0.052 & 6.16 & 0.013 \\
\hline \multicolumn{7}{|l|}{ Paternal indifference } \\
\hline Bipolar disorder v. comorbid & 7.01 & 0.008 & 2.87 & 0.090 & 3.96 & 0.047 \\
\hline Borderline personality disorder $v$. comorbid & 0.70 & 0.401 & 0.02 & 0.879 & 0.01 & 0.922 \\
\hline Bipolar disorder v. borderline personality disorder & 11.70 & 0.001 & 5.83 & 0.016 & 6.35 & 0.012 \\
\hline \multicolumn{7}{|l|}{ Paternal abuse } \\
\hline Bipolar disorder v. comorbid & 12.00 & $<0.001$ & 2.87 & 0.090 & 3.59 & 0.067 \\
\hline Borderline personality disorder $v$. comorbid & 0.40 & 0.527 & 0.00 & 0.982 & 0.01 & 0.928 \\
\hline Bipolar disorder v. borderline personality disorder & 7.04 & 0.008 & 4.77 & 0.029 & 4.39 & 0.036 \\
\hline \multicolumn{7}{|l|}{ Paternal overcontrol } \\
\hline Bipolar disorder v. comorbid & 8.67 & 0.003 & 5.48 & 0.019 & 5.88 & 0.015 \\
\hline Borderline personality disorder v. comorbid & 0.71 & 0.399 & 0.89 & 0.346 & 0.71 & 0.401 \\
\hline Bipolar disorder $v$. borderline personality disorder & 4.19 & 0.041 & 3.60 & 0.058 & 3.66 & 0.056 \\
\hline
\end{tabular}




\begin{tabular}{|c|c|c|c|c|c|c|}
\hline & \multicolumn{2}{|c|}{ DSM diagnosis } & \multicolumn{2}{|c|}{ Clinical strict diagnosis } & \multicolumn{2}{|c|}{ Clinical extended diagnosis } \\
\hline & $\begin{array}{l}\text { Log-odds ratio } \\
\text { (Wald) }\end{array}$ & $P$ & $\begin{array}{l}\text { Log-odds ratio } \\
\text { (Wald) }\end{array}$ & $P$ & $\begin{array}{l}\text { Log-odds ratio } \\
\text { (Wald) }\end{array}$ & $P$ \\
\hline \multicolumn{7}{|l|}{ Total cognitive score } \\
\hline Bipolar disorder v. comorbid & 42.42 & $<0.001$ & 13.02 & $<0.001$ & 18.10 & $<0.001$ \\
\hline Borderline personality disorder $v$. comorbid & 0.27 & 0.605 & 0.00 & 0.988 & 0.19 & 0.663 \\
\hline Bipolar disorder $v$. borderline personality disorder & 40.65 & $<0.001$ & 27.17 & $<0.001$ & 28.76 & $<0.001$ \\
\hline \multicolumn{7}{|l|}{ Suicide attempt } \\
\hline Bipolar disorder v. comorbid & 12.23 & $<0.001$ & 5.89 & 0.015 & 9.42 & 0.002 \\
\hline Borderline personality disorder $v$. comorbid & 0.03 & 0.876 & 1.14 & 0.286 & 1.06 & 0.302 \\
\hline Bipolar disorder $v$. borderline personality disorder & 10.92 & $<0.001$ & 20.35 & $<0.001$ & 24.41 & $<0.001$ \\
\hline \multicolumn{7}{|l|}{ Self-harm attempt } \\
\hline Bipolar disorder v. comorbid & 10.66 & $<0.001$ & 9.90 & 0.002 & 12.34 & $<0.001$ \\
\hline Borderline personality disorder v. comorbid & 0.44 & 0.505 & 1.62 & 0.204 & 2.36 & 0.124 \\
\hline Bipolar disorder $v$. borderline personality disorder & 14.41 & $<0.001$ & 14.52 & $<0.001$ & 16.19 & $<0.001$ \\
\hline
\end{tabular}

disorder group was overrepresented in the unemployed category and underrepresented in full-time employment compared with the bipolar disorder and comorbid groups), age at first depressive episode (higher in the bipolar disorder group), average length of depressive episode (lengthiest in the borderline personality disorder group) and number of self-harm incidents (the comorbid group reporting most incidents followed by the borderline personality disorder and then by the bipolar disorder group). The mean number of DSM listed hypomanic/manic symptoms differed $(F=54.5, P<0.001)$, being lowest for the borderline personality disorder group (3.4) and higher for the bipolar disorder (5.5) and comorbid (6.0) groups.

Tables 1-3 report the log-linear analyses in relation to by clinical extended diagnostic assignment. The overall pattern was again similar to DSM analyses. Specifically, 10 of the 14 bipolar disorder $v$. comorbid comparisons were significant at the $P<0.05$ level, 13 of the 14 bipolar disorder $v$. borderline personality disorder comparisons were significant and, of importance, none of the borderline personality disorder $v$. comorbid comparisons were significant.

\section{Discussion}

Several study limitations and strengths are first noted. Participants were volunteers and aware of our study objectives. Although we included both individuals with type I and II bipolar disorders, the bipolar samples were weighted to those with a bipolar II disorder - where clinical differentiation from a borderline personality disorder is likely to pose greater difficulty than for those with a bipolar I disorder. The relatively few participants with bipolar I disorder disallowed separate analyses of the bipolar I and II subsamples compared with the borderline personality disorder participants and, thus, the extent to which our findings in relation to bipolar status are generalisable to the separate bipolar I and bipolar II conditions cannot be established. Although our principal diagnostic strategy employed strict DSM criteria, we also employed two clinical diagnostic strategies derived by a psychiatrist with clinical experience of both conditions, with those strategies variably respecting and ignoring DSM duration criteria for hypo/mania in those diagnosed with a bipolar disorder. Our set of study variables was broad, capturing clinical features (such as borderline personality style), aetiological factors (such as family history of depression, childhood trauma), condition correlates (such as self-harm attempts) and illness course variables (such as medications trialled).

Although we prioritised DSM diagnostic assignment, the application of two other diagnostic strategies had advantages. First, in demonstrating quite differing rates of assigned diagnoses.
Second, the overall findings generated by the analyses were similar across the differing comparator groups, suggesting that diagnostic nuances such as imposing duration criteria or not for hypo/mania did not compromise the overall model generated. Returning to the first point, even when respecting DSM duration criteria for hypo/ mania, more participants were assigned a clinical diagnosis of a bipolar condition than when respecting DSM diagnoses (an 18\% increase for clinical strict diagnosis and a 49\% increase for clinical extended diagnosis) indicating that the research question risks being influenced by the diagnostic criteria employed for diagnosing bipolar disorder as well as the major impact of factoring in a minimum duration for hypo/manic episodes. The clinical extended bipolar group - comprising those with brief hypo/manic episodes - provided the greatest risk of falsely assigning a borderline personality disorder condition because of their brief episodes of perturbation. However, findings involving the clinical extended group were compatible with comparisons involving the clinical strict bipolar group, again arguing against DSM duration of hypo/manic episodes as being valid requirements of bipolar status as previously overviewed. ${ }^{12}$

DSM decision rules assigned the highest comorbid bipolar disorder/borderline personality disorder rate, which might reflect the greater validity of DSM criteria or, alternately, artefactual comorbidity created by the two conditions sharing some criteria such as emotional dysregulation. The existence of such an artefact is supported by our finding that, while the mean number of DSM hypomanic and manic items was significantly lower in the borderline personality disorder groups (irrespective of diagnostic strategy), those group members reported a mean of three hypomanic or manic symptoms. In their review of the boundaries between borderline personality disorder and bipolar disorder, Zimmerman \& Morgan $^{9}$ reviewed a number of studies to conclude that about $10 \%$ of individuals with borderline personality disorder had a bipolar I disorder and another $10 \%$ a bipolar II disorder, and that $20 \%$ of patients with bipolar II disorder and 10\% with bipolar I disorder were diagnosed with borderline personality disorder - but with there being considerable variation across the individual studies. Their review and our results allow a key second point - that comorbidity is not rare and likely to be more common in those with a bipolar II than a bipolar I disorder. Although our different diagnostic approaches assigned differing subset numbers, they did not clearly impact on key study findings, or challenge the broad findings.

As our principal diagnostic approach respected DSM criteria for each condition we now focus on those analyses. Our three comparison groups had comparable mean ages and did not differ significantly by key socioeconomic variables, so we were not required to control for any sociodemographic impact. The bipolar 
disorder group had fewer women, and lower rates of developmental factors, distinctly lower total borderline personality measure scores and also differed on several treatment variables. Of importance, and against expectation, the three groups did not differ across many variables relating to their depressive history (for example family history of depression, bipolar disorder or suicide, rate and effectiveness of any prescribed antidepressant) or in relation to self-injurious behaviour data, other than the borderline personality disorder group being more likely to have made a suicide or self-harm attempt, with the last being at variance with a study by Zimmerman et $a l,{ }^{17}$ which found that the co-occurrence of the two disorders conferred an additive risk of suicide attempts. Thus, clinical distinction of those with a bipolar disorder, borderline personality disorder or comorbid condition is unlikely to be assisted by clinical information weighting depression variables. The lack of differences in relation to rates of antidepressant use and response to such medication is intriguing and capable of multiple explanations. Such non-specificity is, however, consistent with a parallel finding in a study by Zimmerman et al ${ }^{18}$ reporting that participants with borderline personality disorder with or without a family history of bipolar disorder did not differ in rates of depression, anxiety disorders and suicide attempts, suggesting that such features are more intrinsic to personality nuances rather than any genetically weighted bipolar condition.

Our log-linear analyses compared members of each of the three groups for those variables identified as most clearly differentiating in univariate analyses. In relation to DSM diagnostic assignment, there was a strikingly consistent pattern in that the bipolar disorder group differed significantly from the comorbid group on 13 of the 14 variables examined, the bipolar disorder group differed from the borderline personality disorder group on 12, and the borderline personality disorder and comorbid groups did not differ - or show any trend for differentiation - on any of the 14 variables. Thus, those with a borderline personality disorder diagnosis - alone or as comorbid with a bipolar disorder diagnosis - were more likely to be women, have experienced childhood sexual abuse and other developmental trauma, report depersonalisation in childhood, been recipients of deficient parenting, return strikingly higher borderline personality measure scores and be more likely to have made a self-harm or suicide attempt. Similar patterns - albeit somewhat less distinctive - were returned in relation to the two other diagnostic strategies. Our finding is in line to some degree with a report by Zimmerman et al ${ }^{19}$ identifying that borderline personality disorder presents similarly in those with a bipolar disorder or a major depressive disorder.

\section{Comparison with findings from other studies and interpretation of our findings}

Paris et al ${ }^{20}$ posited four hypotheses regarding the relationship between bipolar disorder and borderline personality disorder. First, borderline personality disorder is a phenotypic variant of bipolar disorder. Second, the converse. Third, that bipolar disorder and borderline personality disorder are independent disorders. Fourth, that each has overlapping aetiologies. They concluded that the third hypothesis was most likely to be valid based on the available evidence, at least in relation to bipolar I disorder as evidence was lacking in relation to bipolar II disorder. Gunderson \& Elliot ${ }^{21}$ and Gunderson \& Phillips ${ }^{22}$ also concluded that, based on family history data, phenomenology of depressive states and response to medication, affective disorders and borderline personality disorder can coexist, but are otherwise unrelated. In another study, Gunderson et $a l^{23}$ concluded that, although bipolar II disorder lengthened the time to remission in those with borderline personality disorder, borderline personality disorder and bipolar disorders were largely independent disorders.
Our current study - both in our earlier comparison of distinct bipolar disorder and borderline personality disorder groups ${ }^{10}$ and the current analyses - also supports a model of two independent conditions and as argued elsewhere by the first author. ${ }^{24}$

Our analyses could be interpreted as indicating that the two conditions may be interdependent (in that no differences were quantified when the borderline personality disorder and the composite borderline personality disorder/bipolar disorder comorbid groups were compared, other than the latter reporting more hypo/manic symptoms). However, comorbidity is formally defined by the coterminous presence of two independent conditions, as we suggest is evidenced by our analyses. If bipolar disorder is positioned within a symptom domain and borderline personality disorder within a personality domain, such a model readily allows that they may occur alone or coterminously. If that model is valid, then clinical differentiation might be reached by adopting a two-tiered diagnostic approach. In essence, assessing whether the patient has a bipolar disorder (weighting bipolar symptoms) or not, a borderline personality disorder (weighting borderline personality features and an antecedent history of developmental difficulties or trauma) or not, or both. In terms of clinical management of individuals with both, the suggested independence model would argue for each condition being treated specifically, presumably involving a mood stabilising medication for the bipolar disorder and psychotherapy for the borderline personality disorder. Model options would include a concurrent management approach or a sequenced approach, and with the latter more likely to prioritise bringing the bipolar disorder under control first to then judge the salience and severity of borderline features.

\section{Limitations}

As noted, our study involved willing volunteers rather than a consecutive sample of relevant patients and our sample had a predominance of those with a bipolar II disorder and relatively few with a bipolar I disorder. Future studies might ensure adequate representation of both bipolar subtypes and undertake similar comparisons of those with a borderline personality disorder against separate type I and type II bipolar disorder subsamples.

\section{Implications}

Study findings allow several conclusions. First, comorbid bipolar and borderline personality disorders are unlikely to be rare, although their prevalence is influenced by diagnostic decision rules. Second, that irrespective of whether borderline personality disorder occurs on its own or in conjunction with a bipolar disorder, its developmental antecedents, personality profile and self-injurious risks correspond and so argue for an independent rather than an interdependent model for comorbid presentations. Third, clinicians should avoid a dichotomous model (i.e. does this patient have a borderline personality disorder or a bipolar disorder) and instead consider whether one or both are present, and then offer separate therapeutic strategies for the identified targeted condition(s).

\footnotetext{
Gordon Parker, MD, PhD, School of Psychiatry and Black Dog Institute, The University of New South Wales, Randwick, Australia; Adam Bayes, FRANZCP, School of Psychiatry, The University of New South Wales, Randwick, Australia; Georgia McClure, BA Psych (Hons), School of Psychiatry and Black Dog Institute, Georgia Mcclure, BA PSych (Hons), School of Psychiatry and Black Dog Institute,
The University of New South Wales, Randwick, Australia; Yolanda Romàn Ruiz del Moral, MPsychiatry, Marina Baixa Hospital, Villijoysa, Spain; Janine Stevenson, PhD, FRANZCP, Westmead Hospital, Sydney, Australia.

Correspondence: Gordon Parker, University of New South Wales and Black Dog Institute, Hospital Road, Randwick, NSW, 2031, Australia. Email: g.parker@unsw.edu.au

First received 2 Nov 2015, final revision 6 Jan 2016, accepted 7 Jan 2016
} 


\section{Funding}

Project Grant Support: NHMRC Program Grant: 1037196.

\section{Acknowledgements}

The authors thank those who assisted with study recruitment and analyses: Kathry Fletcher, Dusan Hadzi-Pavlovic, Vijaya Manicavasagar, Amelia Paterson, Rebecca Graham Stacey McGraw, Rosemary Clancy, Jonathan Levy, Rene de Monchy, Michael Hong, Caryl Barnes, Wayne Borg, Farzaan Mehta, Robert Glassick and Laurie Power.

\section{Appendix}

\section{Individual cognitive items for total personality item score}

1. When I feel irritable or angry, I sometimes hurt myself to relieve stress.

2. I tend to have suicidal thoughts during and after a break-up or being rejected by someone.

3. I do not know who I am really in terms of my identity.

4. I believe I have more difficulties with relationships than the average person my age.

5. I have a big fear of rejection of any kind.

6. If others knew the real me, they would not like me.

7. My value as a person depends enormously on what others think of me.

8. During times of stress, I often feel that others are deliberately mean to me

9. I often feel I have no idea of who I actually am.

10. There have been many times where I have harmed myself.

11. I've felt empty inside for as long as I can remember.

12. I often feel that I have no idea of who I am or any clear identity.

13. I tend to get angry and lose my cool when stressed.

14. I tend to idealise others (i.e. put them on a pedestal) but then often seek to hurt them back if I judge them as hurtful to me.

15. I constantly have intense feelings of personal inadequacy and helplessness.

16. I tend to take things too personally.

17. If someone is critical of something I do, I feel devastated.

18. I think a lot about being deserted by loved ones.

19. If other people knew what I am really like, they would think less of me.

20. I worry about being rejected or abandoned when in a close relationship.

21. I have intentionally harmed myself, for instance by cutting myself or taking too many pills.

22. I often feel like the victim in many situations.

23. I often feel hatred towards someone I care about and need.

24. I tend to lose my temper easily.

25. I can never be really sure if someone approves of me.

\section{References}

1 Zimmerman M, Ellison W, Morgan TA, Young D, Chelminski I, Dalrymple K. Psychosocial morbidity associated with bipolar disorder and borderline personality disorder in psychiatric out-patients: comparative study. Br J Psychiatry 2015; 207: 334-8.

2 Bolton S, Gunderson J. Distinguishing borderline personality disorder from bipolar disorder: differential diagnosis and implications. Am J Psychiatry 1996; 153: 1202-7.
3 Paris J, Gunderson J, Weinberg I. The interface between borderline personality disorder and bipolar spectrum disorders. Compr Psychiatry 2007: 48: 145-54.

4 Paris J. Differential diagnosis of bipolar and borderline personality disorders. Neuropsychiatry 2011; 1: 251-7.

5 Coulston CM, Tanious M, Mulder RT, Porter RJ, Malhi GS. Bordering on bipolar: the overlap between borderline personality and bipolarity. Aust NZ J Psychiatry 2012; 46: 506-21.

6 Bassett D. Borderline personality disorder and bipolar affective disorder. Spectra or spectre? A review. Aust NZ J Psychiatry 2012; 46: 327-39.

7 Mackinnon DF, Pies R. Affective instability as rapid cycling: theoretical and clinical implications for borderline personality and bipolar spectrum disorders. Bipolar Disord 2006; 8: 1-14.

8 Bayes A, Parker G, Fletcher K. Clinical differentiation of bipolar II disorder from borderline personality disorder. Curr Opin Psychiatry 2014; 27: 14-20.

9 Zimmerman M, Morgan TA. Problematic boundaries in the diagnosis of bipolar disorder: the interface with borderline personality disorder. Curr Psychiatry Rep 2013; 15: 422-32.

10 Bayes A, McClure G, Fletcher K, Ruiz Y, Hadzi-Pavlovic D, Stevenson JL, et al. Differentiating the bipolar disorders from borderline personality disorder. Acta Psychiatr Scand 2016; 133: 187-95.

11 Zimmerman M, Martinez JH, Morgan TA, Young D, Chelminski I, Dalrymple K. Distinguishing bipolar II depression from major depressive disorder: demographic, clinical and family history differences. J Clin Psychiatry 2013; 74: 880-6.

12 Parker G, Graham R, Synnott $H$, Anderson J. Is the DSM-5 duration criterion valid for the definition of hypomania? J Affect Disord 2014; 156: 87-91.

13 Parker G, Roussos J, Hadzi-Pavlovic D, Mitchell P, Wilhelm K, Austin MP. The development of a refined measure of dysfunctional parenting and assessment of its relevance in patients with affective disorders. Psychol Med 1997; 27: 1193-203.

14 American Psychiatric Association. Diagnostic and Statistical Manual of Mental Disorder (4th edn) (DSM-IV). APA, 1994

15 Sheehan DV, Lecrubier $Y$, Sheehan $\mathrm{KH}_{\text {, Amorim }} \mathrm{P}$, Janavs J, Weiller $\mathrm{E}$, et al. The Mini-International Neuropsychiatric Interview (M.I.N.I.): the development and validation of a structured diagnostic psychiatric interview for DSM-IV and ICD-10. J Clin Psychiatry 1998; 59 (suppl 20): 22-33.

16 Zanarini MC. The Diagnostic Interview for DSM-IV Personality Disorders. McLean Hospital and Harvard Medical School, 1996.

17 Zimmerman M, Martinez J, Young D, Chelminski I, Morgan TA, Dalrymple K. Comorbid bipolar disorder and borderline personality disorder and history of suicide attempts. J Pers Disord 2014; 28: 358-64.

18 Zimmerman M, Martinez J, Young D, Chelminski I, Dalrymple K. Differences between patients with borderline personality disorder who do and do not have a family history of bipolar disorder. Compr Psychiatry 2014; 55: 1491-7.

19 Zimmerman M, Morgan TA, Young D, Chelminski I, Dalrymple K, Walsh E. Does borderline personality disorder manifest itself differently in patients with bipolar disorder and major depressive disorder? J Pers Disord 2014; 28 : 169-75

20 Paris J, Gunderson J, Weinbeg I. The interface between borderline personality disorder and bipolar spectrum disorders. Compr Psychiatry 2007; 48: 145-54.

21 Gunderson JG, Elliot GR. The interface between borderline personality disorder and affective disorder. Am J Psychiatry 1985; 144: 277-88.

22 Gunderson JG, Phillips KA. A current view of the interface between borderline personality disorder and depression. Am J Psychiatry 1991; 148 967-75.

23 Gunderson JG, Stout RL, Shea MT, Grilo CM, Markowitz JC, Morey LC, et al. Interactions of borderline personality disorder and mood disorders over 10 years. J Clin Psychiatry 2014; 75: 829-34.

24 Parker G. Is borderline personality disorder a mood disorder? Br J Psychiatry 2014; 204: 252-3. 